\title{
NUEVOS REGISTROS EN LA DISTRIBUCIÓN GEOGRÁFICA DE OPHRYACUS SMARAGDINUS (GRÜNWWALD, JONES, FRANZ-CHÁVEZ Y AHUMADA-CARRILLO, 2015)(VIPERIDAE) EN LA REGIÓN DE LAS ALTAS MONTAÑAS, VERACRUZ, MÉXICO
}

\author{
NEW RECORDS ON THE GEOGRAPHIC DISTRIBUTION OF OPHRYACUS SMARAGDINUS (GRÜNWALD, JONES, \\ FRANZ-CHÁVEZ Y AHUMADA-CARRILLO, 2015)(VIPERIDAE) IN THE REGION OF THE HIGH MOUNTAINS, \\ VERACRUZ, MEXICO
}

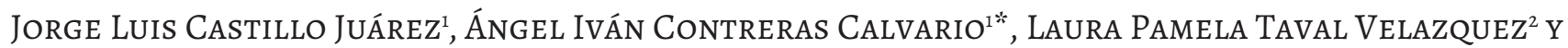 \\ MAURICIO SÁNCHEZ EUGENIO ${ }^{3}$ \\ ${ }^{1}$ Universidad Veracruzana, Facultad de Ciencias Biológicas y Agropecuarias, camino viejo Peñuela-Amatlán de los Reyes. S/n., Mpio. Amatlán de los \\ Reyes, Veracruz, México. CP: 94945. \\ ${ }^{2}$ Fortín de las flores, Colonia San José, México, Veracruz, CP: 94470. \\ ${ }^{3}$ Grupo Ecologista Explorador de Chocaman (GEECH), Chocaman, Veracruz, México, CP: 94160. \\ "Correspondence: acontrerascalvario@gmail.com
}

Este trabajo presenta los primeros registros de Ophryacus smaragdinus en la región de las Altas Montañas (Fig.1), previamente Grünwald et al., 2015 mencionan dos localidades que pertenecen al estado de Veracruz cerca de la región Capital y en la región Huasteca baja, en donde se encuentra la localidad tipo, de igual forma se resalta su distribución restringida para el noreste de Veracruz, desde Huayacocotla hacia el sureste, a través del borde este de la sierra madre oriental, pasando por

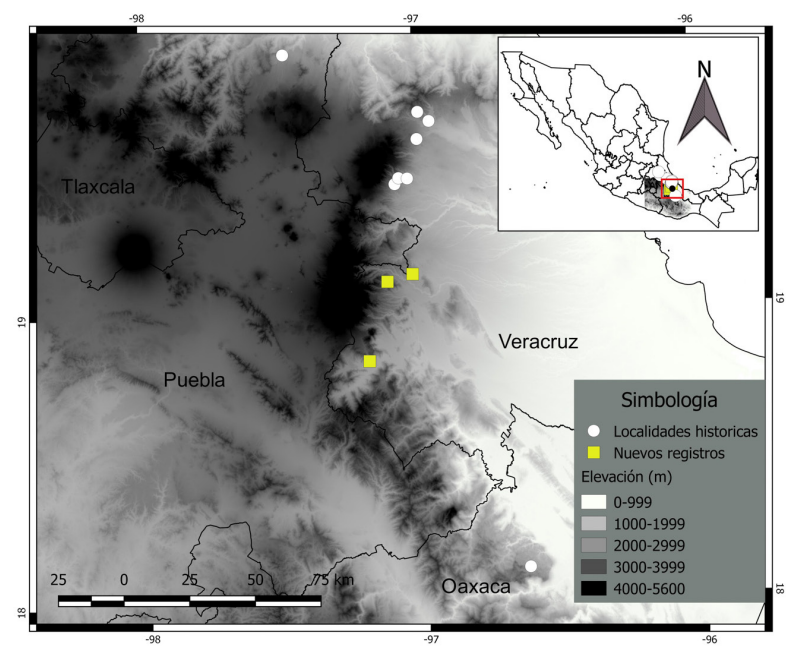

Figure 1. Distribution map of 0 . smaragdinus with historical and new locations. Figura 1. Mapa de distribución de 0. smaragdinus con localidades históricas y nuevas. el centro-este de Hidalgo, noreste de puebla y el centro-oeste de Veracruz. Se menciona la presencia de la especie para sitios cercanos a los municipios de San Andrés Tenejapa y Tequila solo como comunicados personales, sin embargo no existen registros formales publicados para la región montañosa, posteriormente Martinez-Vaca et al., (2016) atribuyen cinco nuevos registros municipales para Veracruz y proponen un modelo de distribución potencial en donde nuestros nuevos registros coinciden con dicho modelo, los ejemplares aquí reportados fueron fotografiados, y las fotografias ingresadas a la Colección Nacional de Anfibios y Reptiles (CNAR).

\section{LOCALIDADES}

México: Veracruz: Municipio de Huatusco $\left(1^{\circ} 07^{\prime} 40.5^{\prime \prime} \mathrm{N}\right.$, 97 ${ }^{\circ} 1^{\prime} 24.8^{\prime \prime} \mathrm{O}$; Datum WGS 84; Elev. 1495 m.) Febrero del 2016. Un ejemplar cría (Fig.2 A (IBH-RF 532) y B.) fue encontrado cruzando una carretera de terracería cerca de las 9:00 horas, la vegetación presente en la zona es bosque mesófilo de montaña en donde sobresale una zona de vegetación riparia, en donde abunda Platanus mexicana Moric y vegetación secundaria.

México: Veracruz: Municipio de Nogales, $\left(18^{\circ} 49^{\prime} 58.50^{\prime \prime} \mathrm{N}\right.$, 97¹1'21.48"O; Datum WGS 84; Elev. 1940 m.) 2 de agosto 2018. Cerca de las 14:00 horas se observó un individuo cría (Fig.3 A (IBH-RF 533) y B.) desplazándose a la orilla de un camino angosto de terracería, el tipo de vegetación del sitio es bosque mesófilo de montaña y vegetación secundaria. 


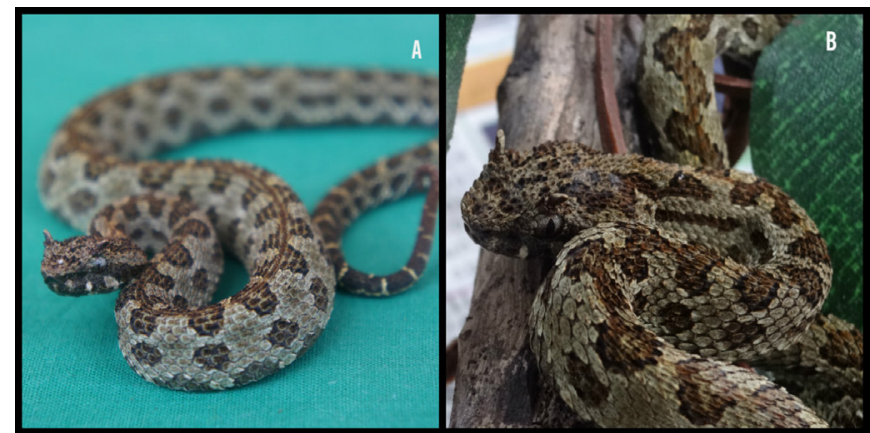

Figure 2. A) Dorsal view of 0 . smaragdinus municipality of Huatusco, Veracruz. B) Approach to appreciate scail detail. Photography: Mauricio Sánchez Eugenio.

Figura 2. A) Vista dorsal de 0. smaragdinus, municipio de Huatusco, Veracruz. B) Acercamiento para apreciar detalles de escamación. Fotografías: Mauricio Sánchez Eugenio.

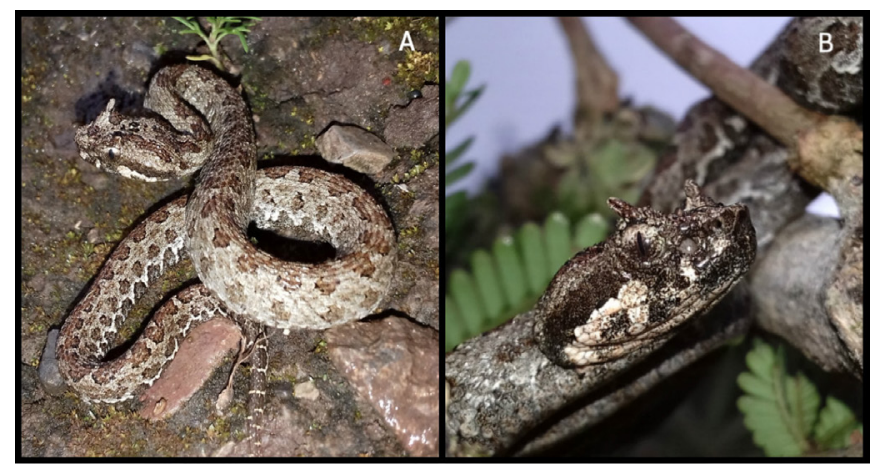

Figure 3. A) Dorsal view of 0 . smaragdinus municipality of Nogales, Veracruz. B) Approach to appreciate scail detail. Photography: Ángel Iván Contreras Calvario.

Figura 3. A) Vista dorsal de 0. smaragdinus, municipio de Nogales, Veracruz. B) Acercamiento para apreciar detalles de escamación. Fotografías: Ángel Iván Contreras Calvario.

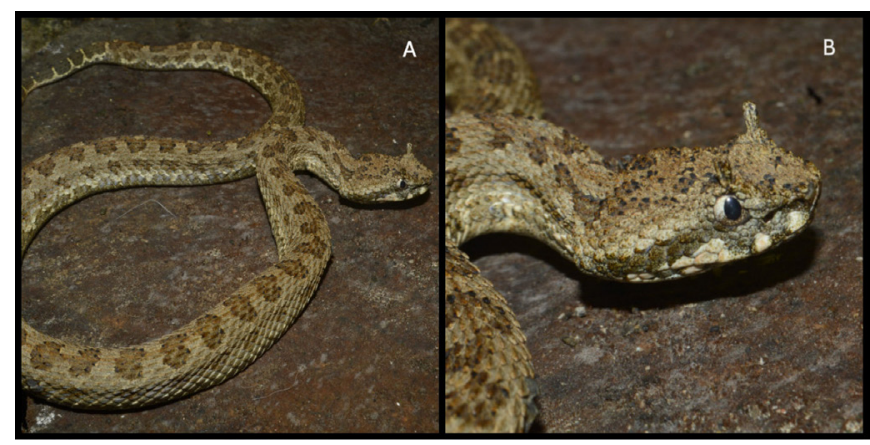

Figure 4. A) Dorsal view of 0 . smaragdinus municipality of Alpatlahuac, Veracruz. B) Approach to appreciate scail detail. Photography: Jorge Luis Castillo Juárez.

Figura 4. A) Vista dorsal de 0. smaragdinus, municipio de Alpatlahuac, Veracruz. B) Acercamiento para apreciar detalles de escamación. Fotografías: Jorge Luis Castillo Juárez.
México: Veracruz: Municipio de Alpatlahuac, (19 ${ }^{\circ} 6^{\prime} 10.75^{\prime \prime} \mathrm{N}$, $97^{\circ}$ 6'23.45"O; Datum WGS 84; Elev. 1915 m.) 25 de Agosto 2018. Cerca de las 13:00 horas se observó un individuo juvenil (Fig.4 A (IBH-RF 534) y B.) asoleándose cerca de un cuerpo de agua, el tipo de vegetación de la zona es bosque mesófilo de montaña y vegetación secundaria. Los registros de $\mathrm{O}$. smaragdinus que fueron previamente reportados según la literatura los añadimos a una tabla en donde presentamos nuestras nuevas localidades para la distribución de la especie (Tabla 1).

Table 1. Historical and new records in the distribution of Ophryacus smaragdinus. Table 1. Registros históricos y nuevos en la distribución de Ophryacus smaragdinus.

\begin{tabular}{cccc}
\hline Registros & Lat (N) & Lon (W) & Localidad \\
\hline Históricos & 20.3149 & -98.2296 & Tenango de doria, Hidalgo \\
Históricos & 18.1081 & -96.6339 & San José Tenango, Oaxaca \\
Históricos & 19.8957 & -97.4717 & Tlatlauquitepec, Puebla \\
Históricos & 19.4395 & -97.0780 & Xico, Veracruz \\
Históricos & 20.6557 & -98.4248 & Huayacocotla, Veracruz \\
Históricos & 19.4598 & -97.0633 & Xico viejo, Veracruz \\
Históricos & 19.4576 & -97.0318 & Micoxtla, Veracruz \\
Históricos & 19.6532 & -96.9465 & Coacoatzintla, Veracruz \\
Históricos & 19.6858 & -96.9863 & Etlantepec, Veracruz \\
Históricos & 19.5924 & -96.9925 & San Miguel del soldado \\
Nuevos & 18.8329 & -97.1893 & Nogales, Veracruz \\
Nuevos & 19.1029 & -97.0898 & Alpatlahuac, Veracruz \\
Nuevos & 19.1279 & -97.0235 & Huatusco, Veracruz \\
\hline
\end{tabular}

Nuestra contribución aporta tres nuevos registros que sumados a los anteriormente mencionados, da como resultado diez municipios en los que se distribuye Ophryacus smaragdinus para el estado de Veracruz, se amplía la distribución de la especie $72 \mathrm{~km}$ en línea recta desde el punto más cercano conocido (Xico, Veracruz), hacia el más sureño aportado en este trabajo (Nogales, Veracruz). Se reporta la presencia de la especie en el Parque Nacional Cañón del Río Blanco, el cual representa el único refugio natural en Veracruz, ya que el municipio de Nogales se encuentra dentro del polígono de dicha ANP. Consideramos que al tratarse de una especie recientemente descrita, la poca información sobre su historia natural y que según Grünwald et al., 2015 está catalogada por medio de EVS como en "alta vulnerabilidad", nuestros aportes contribuyen al conocimiento 
de dicha especie y por esto se deben plantear futuras estrategias para su conservación y proponer acciones inmediatas para evitar el deterioro de sus hábitats.

\section{LITERATURA CITADA}

Grünwald, C.I., J.M. Jones, H. Franz-Chávez \& I. AhumadaCarrillo. 2015. A new species of Ophryacus (Serpentes: Viperidae: Crotalinae) from eastern Mexico, with comments on the taxonomy of related pitvipers. Mesoamerican Herpetology 2: 388-416.

Martínez-Vaca León, O. I., E.A. Bello-Sánchez \& J.E. Morales-Mávil. 2016. New distributional records of the Emerald Horned Pitviper Ophryacus smaragdinus, in central Veracruz. Acta Zoológica Mexicana (n.s.) 32(3):393-397. 